\title{
Arcuate Plan of Chick Midbrain Development
}

\author{
Timothy A. Sanders, ${ }^{1}$ Andrew Lumsden, ${ }^{2}$ and Clifton W. Ragsdale ${ }^{1}$ \\ ${ }^{1}$ Department of Neurobiology, Pharmacology, and Physiology, The University of Chicago, Chicago, Illinois 60637, and \\ 2The Medical Research Council Centre for Developmental Neurobiology, King's College London, Guy's Hospital, London \\ SE1 1UL, United Kingdom
}

In spinal cord and hindbrain development, neurons are generated as longitudinal cell columns aligned with the ventral and dorsal midlines. For rostral brain, however, the fundamental structure of early neuronal patterning remains poorly understood. We report here that, in the chick embryo, the ventral midbrain is remarkably regular in its cellular and molecular organization; it is arranged as a reiterative series of arcuate territories arrayed bilateral to the ventral midline. In the mantle layer of the ventral midbrain, an arcuate series of neuronal cell columns (midbrain arcs) is demonstrated by acetylcholinesterase histochemistry and gene expression for class III $\beta$-tubulin, homeodomain transcription factors, and neurotransmitter synthetic enzymes. In the ventricular layer of midbrain progenitor cells, WNT and NOTCH ligand gene expression displays arcuate periodicities that form a tight three-dimensional registration with the arcs of the underlying mantle layer. Ventral midbrain arcuate patterning is even macroscopically visible, forming ridges along the ventricular surface. These observations establish that a single plan of arcuate organization governs the morphogenesis and cell-type specification of the ventral midbrain. Arcs are not restricted to the midbrain tegmentum but extend through the subthalamic tegmentum of the forebrain. Thus, the chick rostral brain, which is classically divided into midbrain and forebrain, can also be partitioned into the following: (1) a neuraxial region of arcs and (2) an anterodorsal cap that includes midbrain tectum and nonsubthalamic forebrain. We show that this partition of brain tissue is supported by the expression patterns of homologs of Drosophila gap genes.

Key words: midbrain arcs; acetylcholinesterase; oculomotor neurons; nucleogenesis; NOTCH; WNT5A; Tailless
Despite their complete dissimilarity in tissue cross sections, the spinal cord and hindbrain share a common plan of organization (Johnston, 1906; Herrick, 1915). In spinal cord, motor neurons and secondary sensory neurons are arrayed into interrupted longitudinal cell columns, with motor neuron columns placed ventrally and laterally and sensory relay columns situated dorsally. In hindbrain, this basic plan is elaborated by the addition of motor columns to accommodate the branchial arches and sensory columns for the sensory ganglia of the head. There is good evidence that this columnar design is laid down early in vertebrate neurogenesis. Neuroembryologists beginning with His (1904) have described a longitudinal sulcus limitans in spinal cord and hindbrain that sets off a basal plate, containing motor neurons, from an alar plate, enriched in secondary sensory neurons (Sidman and Rakic, 1982). Within these embryonic plates, frank longitudinal cell columns have long been recognized with Nissl staining and enzyme histochemistry (Herrick, 1915; Hugosson, 1958; Kallen, 1962). More recently, direct demonstration that motor neurons, secondary sensory neurons, and neuronal differentiation control

\footnotetext{
Received May 8, 2002; revised Sept. 18, 2002; accepted Sept. 18, 2002.

This work was supported by a March of Dimes Basil O'Connor award (C.W.R.), a Medical Research Council Programme grant (A.L.), and grants from the National Institute of Neurological Disorders and Stroke/National Institutes of Health (C.W.R.) and the Wellcome Trust (A.L., C.W.R.). We thank A. Brown, J.-F. Brunet, D. Cleveland, C. Goridis, M. Goulding, A. Graham, H. Hayashi, D. Henrique, D. Ish-Horowicz, T. Jessell, J. Lewis, G. Martin, A. Myat, T. Reh, C. Tabin, M. Wassef, D. Wu, and R. Yu for cDNAs and Seema Agarwala, Ken Brady, and Sue Lundy for their help.

Correspondence should be addressed to Clifton W. Ragsdale, Department Neurobiology, Pharmacology, and Physiology, The University of Chicago, 947 East 58th Street, Chicago, IL 60637. E-mail: cliff@drugs.bsd.uchicago.edu.

Copyright (C) 2002 Society for Neuroscience 0270-6474/02/2210742-09\$15.00/0
}

genes are arrayed into multiple longitudinal columns in early spinal cord and hindbrain development has been provided in molecular studies identifying markers of cell-type identity and developmental regulatory genes (Tsuchida et al., 1994; Chitnis et al., 1995; Myat et al., 1996; Logan et al., 1998).

The fundamental design principles for rostral brain are much less clear. Columns of secondary sensory neurons end in rostral hindbrain, and motor neurons extend only to the oculomotor complex of the midbrain. Moreover, in adult midbrain and forebrain, only a very few nuclei form frank longitudinal cell columns, and the rest appear too variegated in shape to suggest a simple mechanism of histogenesis (Nauta and Feirtag, 1986). A starting point for many studies of rostral brain embryology has been the suggestion of His (1904) that a sulcus limitans defining alar and basal plates can be traced to the preoptic recess in rostral forebrain. This trajectory, which would provide for broad longitudinal zones in rostral brain, has attracted some support recently (Puelles et al., 1987; Shimamura et al., 1995). Many workers, however, have placed the termination of the sulcus limitans more caudally, near the mammillary recess (Schulte and Tilney, 1915; Kingsbury, 1922; Coggeshall, 1964; Kuhlenbeck, 1973), or have challenged whether the sulcus can be followed into forebrain at all (Johnston, 1923; Rose, 1942). In embryonic hindbrain and spinal cord, it is not the sulcus limitans, although that provides the most compelling morphological evidence for a longitudinal organization. It is the organization of neurons into distinct longitudinal columns. For this reason, the starting point of our study was an examination of the mantle layer of postmitotic neurons during midbrain and forebrain embryogenesis.

We began by staining chick brain whole mounts for acetylcholinesterase (AChE) activity. For studies of adult brain anatomy, 
AChE histochemistry rivals Nissl staining as a method for demonstrating brain nuclei in tissue sections (Graybiel and Ragsdale, 1978; Paxinos and Watson, 1986). AChE staining has also been used to identify early patterns of postmitotic neurons in CNS development (Layer, 1983; Moody and Stein, 1988; Ross et al., 1992). Unexpectedly, we found that AChE staining of embryonic brain whole mounts demonstrates an extremely regular pattern in the mantle layer of the ventral midbrain. It is organized into arcuate stripes. We describe here the anatomy of these stripes, which we call midbrain arcs. We demonstrate that the arcs differ in the cell types they contain. For example, the motor neurons of the oculomotor complex are restricted to the most medial arc, and more lateral arcuate territories are enriched in the synthetic enzyme for the neurotransmitter GABA. We show that an arcuate organization is also present in midbrain ventricular layer of progenitor cells and that these arcuate patterns lie in precise radial alignment with those of the mantle layer. We demonstrate that the arcuate organization of the ventral midbrain is even visible in unstained brain tissue. Together, our findings establish that the fundamental plan of the embryonic ventral midbrain is a quite simple pattern: a series of arcuate territories aligned with the longitudinal axis of the brain.

\section{MATERIALS AND METHODS}

Tissue preparation. Fertilized chicken eggs were placed for $2-8 \mathrm{~d}$ in a humidified forced-draft incubator set at $37.5^{\circ} \mathrm{C}$. Embryos collected for histochemistry were submerged in $4 \%$ paraformaldehyde in a PBS solution. Cold $2.5 \%$ glutaraldehyde in PBS was used as a fixative for the scanning electron microscopy specimens. Embryo staging followed Hamburger and Hamilton (1951).

cDNAs for riboprobe generation. An 849 bp PCR product identifying chick glutamate decarboxylase (GAD2) (GenBank accession number AF317501; 84\% nucleotide and $94 \%$ amino acid identity with the rat 65 $\mathrm{kDa}$ glutamate decarboxylase gene) was amplified from embryonic day 5 (E5) chick brain cDNA with forward primer 5'-RAC DGC MAA YAC BAA YAT GTT YAC-3' and reverse primer $5^{\prime}$-GCT GGG TTT GAG ATG ACC ATC C-3'. The GAD2 fragment was subcloned into pCRII (Invitrogen, San Diego, CA), and riboprobes were generated with SP6 polymerase incubation of NotI-digested phagemid. $A C H E$ riboprobes were derived from a 472 bp PCR fragment amplified from chicken genomic DNA and corresponding to bases 298-769 of GenBank accession number U03472 [T7 polymerase runoffs of an EagI-digested Bluescript $\mathrm{SK}(+)$ subclone]. Class III $\beta$-tubulin gene expression was detected using a 434 bp BstZ1-SmaI subclone of the chicken $\beta_{4}$-tubulin genomic clone $\mathrm{p} \beta \mathrm{G} 4$ (Lopata et al., 1983). This fragment contains an isotypespecific sequence representing the $230 \mathrm{bp} 3^{\prime}$ end of the transcript, and riboprobes were generated by T7 transcription of an EagI-digested Bluescript SK(+) subclone. Riboprobes were also synthesized from vectors harboring chick cDNAs for CASH1/ASCL1 (1.9 kb) (Jasoni et al., 1994), choline acetyltransferase (CHAT) $(2.5 \mathrm{~kb})$ (Yamada et al., 1993), DELTA1/DLL1 (700 bp) (Myat et al., 1996), EVX1 (1 kb) (M. Dush and G. Martin, unpublished cDNA), NOTCH1 (1 kb) (Myat et al., 1996), OTX2 (1.5 kb) (Bally-Cuif et al., 1995), PAX3 (660 bp) and PAX6 (540 bp) (Goulding et al., 1993), PHOX2A/ARIX (430 bp) (Ernsberger et al., 1995), SERRATE1 (900 bp) (Myat et al., 1996), SERRATE2 (1.6 kb) (Hayashi et al., 1996), TAILLESS/NR2E1 (1.3 kb) (Yu et al., 1994), $W N T 5 A$ (1.2 kb subclone), and $W N T 7 A$ (1.2 kb subclone) (Dealy et al., 1993).

Histochemistry. AChE staining was done on whole-mount preparations with a two-step modification of the copper thiocholine method (Graybiel and Ragsdale, 1978). High-stringency whole mount in situ hybridization was performed with riboprobes labeled with the haptens digoxigenin and fluorescein. Labeled RNA duplexes were detected with antibodyphosphatase conjugates (Roche Molecular Biochemicals, Indianapolis, IN), and phosphatase activity was demonstrated with the distinguishable tetrazolium substrates nitro blue tetrazolium and tetranitro blue tetrazolium (Grove et al., 1998).

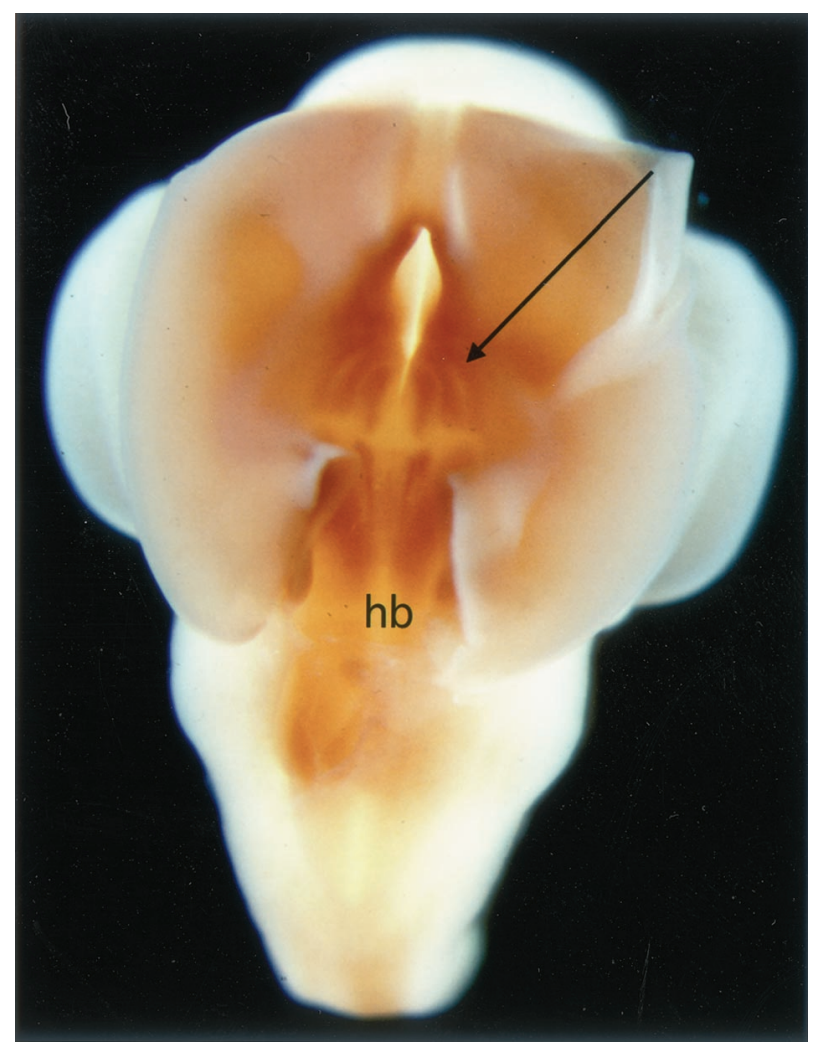

Figure 1. Arcs of the embryonic midbrain tegmentum are defined by AChE histochemistry. Whole-mount AChE staining of stage 28 (E6) chick embryo head reveals stripes (arcs) of dense enzyme activity in ventral midbrain. The back of the head, including the midbrain tectum and rostral roof of the hindbrain, was cutoff before staining. Caudal is to the bottom of the figure; rostral structures, including the forebrain, are in the $z$-axis away from the viewer. Arrow marks one of the arcs. $h b$, Hindbrain.

\section{RESULTS}

\section{The midbrain arcs are defined by acetylcholinesterase histochemistry}

To study pattern formation in early midbrain development, we stained chick embryo whole mounts for AChE activity (Fig. 1). Beginning on E3, AChE staining detects stripes in the midbrain tegmentum (ventral midbrain). By E5 (stage 26), four well defined AChE-rich stripes, 80-160 $\mu \mathrm{m}$ across, are readily identified bilateral to the ventral midline (Fig. $2 A$ ). By E6, a fifth, lateralmost AChE-dense stripe is also evident (data not illustrated). At E5, the AChE-poor tissue between the stripes is narrow, except between the third and fourth most lateral AChE-rich stripes, which are well separated caudally. By E7, the other lateral AChEpoor interstripes have broadened as well. The AChE-rich and -poor stripes mark out columns of cells in the mantle layer of the neural tube, in which postmitotic neurons accumulate (Fig. $3 A$ ). Because of the arcuate shape of these columns, which is accentuated by the curve of the cephalic flexure, we give the AChE-rich stripes the name midbrain arcs, and we refer to the AChE-poor stripes as interarcs. The arcs are a transient feature of midbrain development, conspicuous in whole-mount preparations until stage 30 (E7), but are then obscured as tegmentum growth continues.

In studies of embryonic brain anatomy, AChE activity has been proposed to serve as a general marker of postmitotic neurons 
Figure 2. Arcs of the midbrain tegmentum form functionally and molecularly distinguished territories. $A$, Whole-mount in situ hybridization of $A C H E$ gene expression in midbrain tegmentum identifies arcs $1-4$, numbered medial to lateral from the ventral midline. The orientation is as in Figure 1, and the view is from the ventricular surface. The ventral midbrain and adjoining hindbrain have been dissected away from the forebrain and the other head tissues of stage 26 (E5) chick embryos and flattened on a glass slide with a coverslip. The midbrain-hindbrain junction [isthmus (is)] and the ventral midline, including the floor plate $(f p)$ of hindbrain, are unstained. $B$, Arcs are also demonstrated by gene expression for class III $\beta$-tubulin $(\beta T U B)$, an established
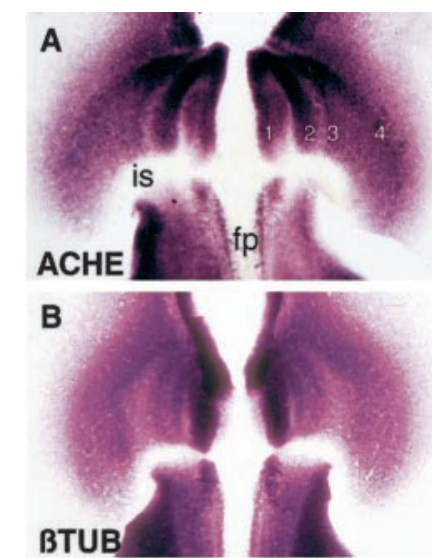
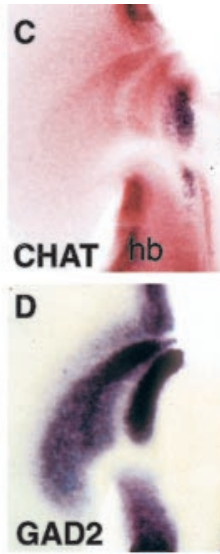
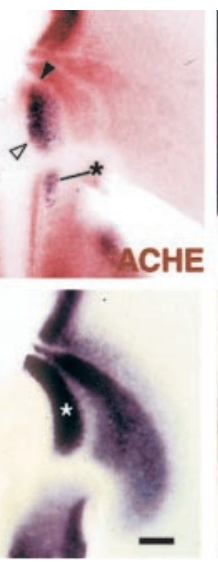
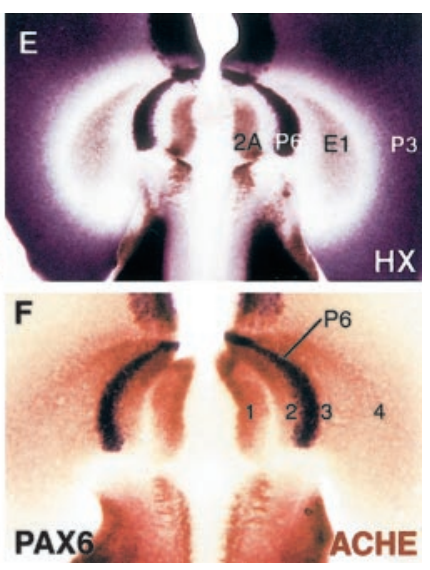

marker of postmitotic neurons. $C$, Two-color in situ hybridization experiment establishes that CHAT-positive motor neurons (blue, marked by open arrowhead) are confined to the first arc. Arcs demonstrated by $A C H E$ gene expression appear red. Filled arrowhead notes the rostral limit of midbrain tegmentum. Asterisk indicates CHAT-rich trochlear motor neurons in the hindbrain $(h b) . D, G A D 2$ gene expression marks out arc 2 (asterisk) and the more lateral arcs and interarcs but is not detected in the medial arc. $E$, Two-color, four-probe detection of regularly spaced arcuate territories of homeobox $(H X)$ gene expression in ventral midbrain. 2A, PHOX2A; P6, PAX6; E1, EVX1; P3, PAX3. F, Two-color in situ hybridization demonstration that the PAX6-rich stripe (P6) lies in the $A C H E$-poor interarc between $\operatorname{arcs} 2$ and 3. Scale bar: $A, C, F, 200 \mu \mathrm{m} ; B, D, 225 \mu \mathrm{m} ; E, 250 \mu \mathrm{m}$.

(Layer, 1983; Moody and Stein, 1988; Ross et al., 1992). Our findings with a second, specific neuronal marker, class III $\beta$-tubulin (Moody et al., 1989; Lee et al., 1990; Easter et al., 1993; Menezes and Luskin, 1994), support this view. The pattern of gene expression for $\beta I I I$-tubulin in chick embryonic ventral midbrain is strikingly similar to that for $A C H E$ and clearly demonstrates arcs (Fig. $2 A, B$ ). These observations establish that the midbrain arcs are neuronal structures and that the interarcs, as well as the arcs, contain postmitotic neurons.

\section{The midbrain arcs have distinct neurotransmitter and transcription factor identities}

To assess the relationship of the AChE-rich arcs to the motor neurons of the midbrain, we performed correlative anatomy experiments marking the midbrain oculomotor neurons by gene expression for $C H A T$ (Fig. $2 C$ ) and by retrograde labeling of third cranial nerve axons (data not shown). These experiments established that the neurons of the oculomotor nucleus have a precise relationship to the midbrain arcs; they are restricted to the first arc. In contrast, cells expressing the GABA synthetic enzyme $G A D$ are not found in the first arc but are enriched in the lateral arcs (Fig. 2D, GAD2/GAD65; GAD1/GAD67, data not illustrated).

Transcription factor gene expression also demonstrates that the midbrain arcs differ in their molecular identities. Our most striking findings are for a panel of homeobox genes that together identify precise, regularly spaced arcuate domains within the mantle layer of midbrain tegmentum (Agarwala et al., 2001) (Fig. $2 E, F)$. The homeobox gene $P H O X 2 A$, which is thought to be expressed by oculomotor neurons (Pattyn et al., 1997), marks the first arc; the homeobox gene $P A X 6$ serves to label the interarc between arcs 2 and 3 ; the homeobox gene $E V X 1$ distinguishes the $3 / 4$ interarc and arc 4; and the homeobox gene $P A X 3$, which is expressed throughout the ventricular layer of midbrain tectum (Goulding et al., 1991), identifies the lateral edge of the midbrain tegmentum. The sequential onsets of tegmental expression for $P H O X 2 A$ (by stage 12), PAX6 (stage 19), and EVX1 (stage 23) suggest that the unique molecular identities of the midbrain arcs may generally develop in a medial-to-lateral progression.

Together, these findings with neurotransmitter-specific and homeobox gene expression suggest that the midbrain arcs identify a patterning system by which functionally and molecularly distinct assemblies of midbrain neurons are formed in early development.

\section{Periodicities in the midbrain ventricular layer}

The acetylcholinesterase-rich arcs are restricted to the mantle layer of postmitotic neurons (Fig. $3 A$ ), but arcuate patterns in midbrain gene expression are not. In the ventricular layer of tegmental progenitor cells, there are arcuate heterogeneities in gene expression for components of the NOTCH and WNT signaling pathways (Figs. $3 B-F, 4$ ). The NOTCH ligand DELTA1 is distributed in a series of arcuate bands arrayed bilateral to the ventral midline (Fig. 3B). Similar arcuate territories are identified in whole-mount preparations for the related proneural gene $C A S H 1 / A S C L 1$ (Fig. 3E,F). In the embryonic hindbrain, DELTA1 gene expression demonstrates longitudinal bands that interdigitate with stripes of messages for a second NOTCH ligand, SERRATE1 (Myat et al., 1996). We found a similar complement in ventral midbrain. SERRATE1 and SERRATE2 mRNAs are expressed in arcuate bands (Fig. $3 B, C$ ), and these fall within the periodic gaps in DELTA1 expression (correlation not documented).

Of the three WNT genes reported to be expressed in developing mouse ventral midbrain (Parr et al., 1993), one of them, $W N T 1$, is restricted to the midbrain-hindbrain junction during chick tegmentum embryogenesis (Fig. 4A) (Bally-Cuif and Wassef, 1994). The other two, however, are expressed in developing ventral midbrain, in which they form clear arcuate periodicities (Fig. 4B,C). WNT5A is expressed in a strikingly regular pattern of three uniformly spaced stripes bilateral to the ventral midline (Fig. 4B). The distribution of $W N T 7 A$ message is more complex but is also arranged into arcuate periodicities (Fig. 4C).

We tested in two-color, whole-mount in situ hybridization experiments how the periodicities in progenitor layer signaling molecules relate to the pattern of arcs and interarcs in midbrain mantle layer. These correlative experiments established a strict three-dimensional registration between gene expression patterns in the two embryonic layers (Figs. $3 E, F, 4 D-F$ ). The precision of this registration is best illustrated by the interdigitation of the ventricular layer $W N T 5 A$ bands with the mantle layer homeobox genes $P H O X 2 A, P A X 6$, and $E V X 1$, when viewed in whole-mount midbrain preparations. The medial gap in $W N T 5 A$ expression 

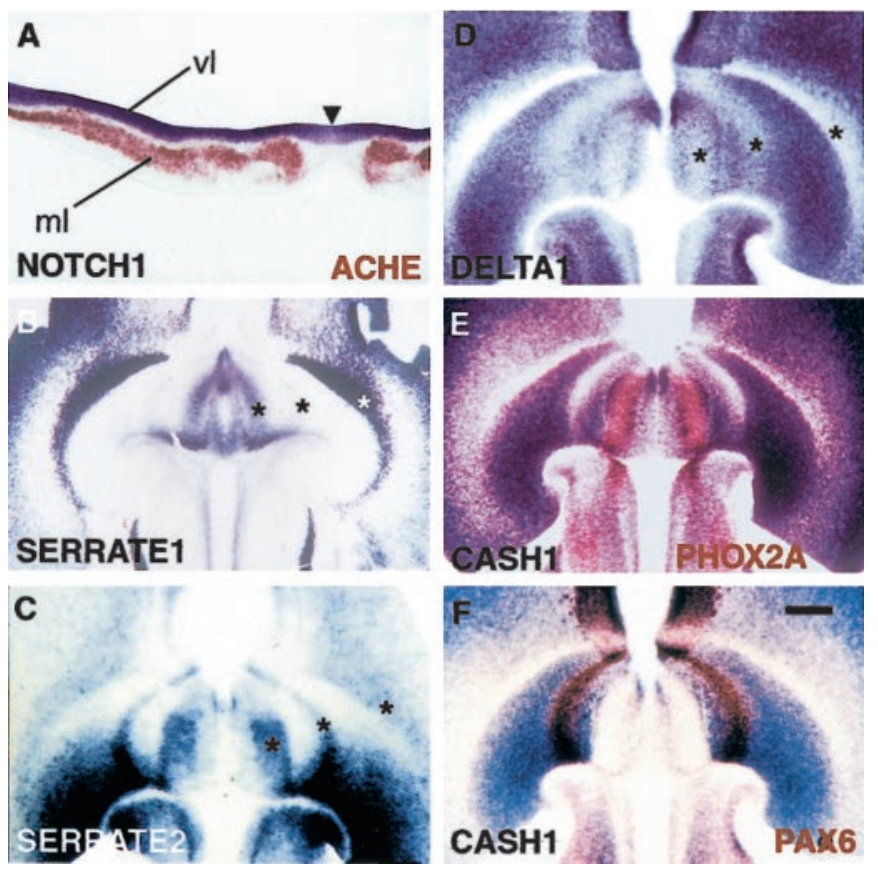

Figure 3. Gene expression for NOTCH signaling components identifies stripes in midbrain ventricular layer. $A$, Cross section through ventral midbrain illustrating the mantle $(\mathrm{ml})$ and ventricular $(v l)$ layers, which are readily distinguished by $A C H E(\mathrm{ml})$ and $\mathrm{NOTCH} 1(\mathrm{vl})$ gene expression. Note that $A C H E$-rich arcs are less conspicuous in tissue sections than in whole-mount preparations. Arrowhead indicates ventral midline. $B, C$, Whole-mount gene expression for the NOTCH ligands SERRATE1 (B) and SERRATE2 $(C)$ demonstrates ventricular layer stripes. $D$, A series of ventricular layer arcuate bands bilateral to the ventral midline is seen with in situ hybridization for the NOTCH ligand DELTA1. Asterisks mark DELTA1 mRNA-poor zones. Comparison of the fiducial asterisks in $B-D$ illustrates that the SERRATE and DELTA1 patterns interdigitate, with the lateral gap in DELTA1 expression being particularly dense in SER$R A T E 1$ message and the two more medial DELTA1-poor zones being enriched in SERRATE2 mRNA. E, $F$, Two-color whole mounts document that the arcuate bands in the mantle and ventricular layers lie in spatial register. In these experiments, the whole mounts serve to "project" the expression pattern of one embryonic layer onto another. Despite the distortions created by flattening, which include some shear between the ventricular and mantle layers, the interdigitation between the mantle layer homeobox genes PHOX2A (E, brown) and PAX6 (F, brown) and the ventricular layer proneural gene $C A S H 1$ (blue) is clear. At this age, $C A S H 1$ gene expression closely follows that of DELTA1 (see D). Orientation of E5 whole mounts $(B-F)$ as in Figure 2. Scale bar: $A, C, D, 300$ $\mu \mathrm{m} ; B, 400 \mu \mathrm{m} ; E, 350 \mu \mathrm{m} ; F, 325 \mu \mathrm{m}$.

overlies the $P H O X 2 A$-positive first arc (Fig. $4 D$ ), the lateral $W N T 5 A$ stripes straddle the $P A X 6$-rich interarc (Fig. $4 E$ ), and the lateral edge of $W N T 5 A$ expression is hugged by the EVX1 band (Fig. $4 F$ ). The schematic diagram of Figure 5 summarizes this spatial architecture for the ventral midbrain, describing the relationship of the $A C H E$-rich arcs and the homeobox gene territories of the mantle layer to the $W N T 5 A$ and DELTA1 periodicities of the ventricular layer.

\section{Morphological demonstration of the midbrain arcs}

We found that histochemistry is not required to demonstrate the arcuate patterning of the ventral midbrain. Arcuate periodicities are visible in freshly dissected, unstained midbrain whole mounts (Fig. 6A). At least part of these refractive variations may be caused by a series of delicate ridges that furrow the ventricular surface of the ventral midbrain (illustrated in the scanning electron photomicrograph of Fig. $6 B$ ). These ridges, which are visible
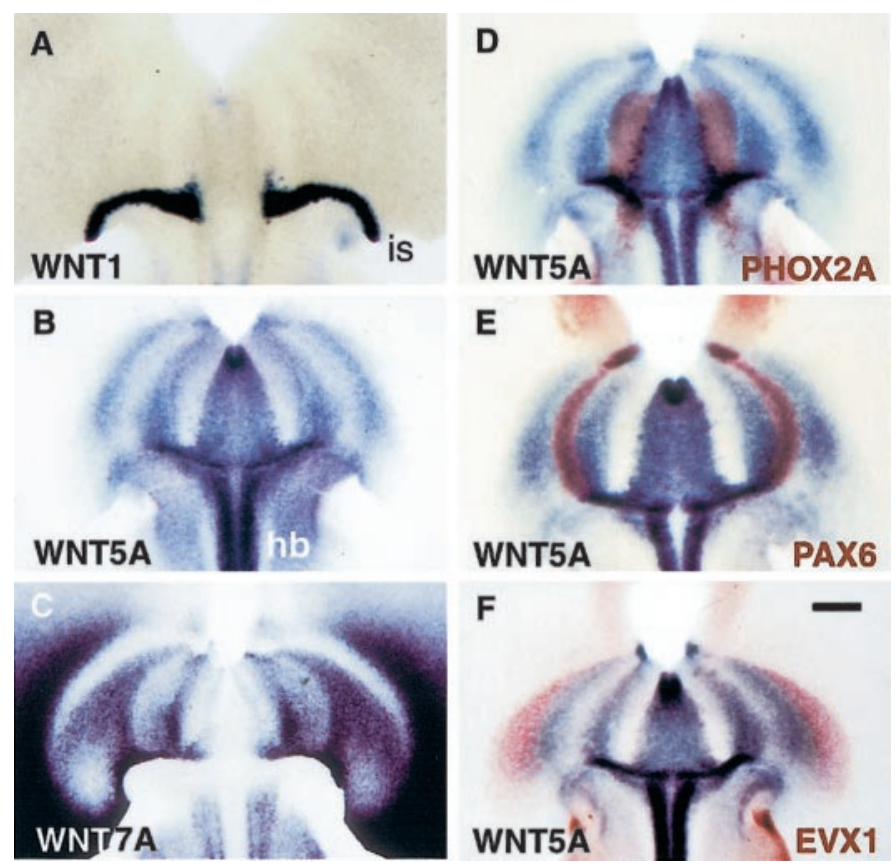

Figure 4. Periodic patterns of $W N T$ gene expression demonstrated in midbrain ventricular layer. $A, W N T 1$ message in the E5 chick is detected at the isthmus but not in the ventral midbrain. $B, C, W N T 5 A(B)$ and $W N T 7 A(C)$ mRNAs are expressed in the ventral midbrain ventricular layer, in which they form periodic arcuate bands. $D-F$, The WNT5A periodicities interdigitate with the homeobox genes expressed in the mantle layer when viewed in whole-mount preparations. Two-color in situ hybridization experiments detect $W N T 5 A$ expression in blue and $P H O X 2 A(D), P A X 6(E)$, and $E V X 1(F)$ expression in brown. Midbrain whole mounts at stage $26(A, B, D-F)$ and stage $27(C)$, oriented as in Figure 2. $h b$, Hindbrain; is, isthmus. Scale bar: $A, 175 \mu \mathrm{m} ; B, D, E, 200$ $\mu \mathrm{m} ; C, 275 \mu \mathrm{m} ; F, 230 \mu \mathrm{m}$.

beginning at approximately stage 22 and persist through stage 30 , overlie the AChE-rich arcs of the mantle layer (correlation not illustrated).

\section{The relationship of the arcs to domains of gap gene expression}

By AChE staining, the midbrain arcs extend in reduced numbers into the rostrally adjoining subthalamic region of the forebrain (Fig. 7A). There, at the level of the intrathalamic zona limitans (zli), the medial subthalamic arc abruptly terminates, and the lateral arcs appear to be deflected dorsally along the posterior border of the zli. A similar trajectory is observed in the patterns of ventricular layer developmental control genes (Fig. 7, B, $C A S H 1, C, O T X 2)$. These observations suggest a division of rostral brain into a posteroventral domain containing arcs aligned with the ventral midline (embryonic midbrain and subthalamic tegmentum) and an anterodorsal domain comprising midbrain tectum and nonsubthalamic forebrain. This division of rostral brain falls orthogonal to the transverse boundary between midbrain and forebrain, which is taken to be primary in current models of forebrain development (Shimamura et al., 1995). If the arc-based division of rostral brain is also fundamental, then it may be reflected in the expression patterns of developmental control genes involved in brain regionalization.

Molecular genetic studies of Drosophila development have identified a small set of terminal and head gap genes that control the development of broad overlapping domains of the fly brain (Cohen and Jurgens, 1990; Finkelstein and Perrimon, 1991; Hirth 
Figure 5. Schematic summary of the three-dimensional spatial registrations in ventral midbrain among the developmental signaling molecules (WNT5A,DELTA1) of the ventricular layer (top tier) and the arcs $(A C H E)$ and the homeobox genes $(H X)$ of the mantle layer (bottom tier). Arcs are numbered $1-5$ on both sides of the ventral midline. $H X$ genes, Compendium of expression patterns for homeobox genes $P H O X 2 A, P A X 6, E V X 1$, and $P A X 3$, ordered sequentially from the midline as in Figure $2 E$ and distinguished by hue. Expression throughout the midbrain tectum of DELTA1 and the $H X$ gene $P A X 3$ is indicated by arrows, the base of which identifies the lateral edge of the tegmentum. This summary is based on pairwise comparisons of gene expression in two-color in situ hybridization whole-mount preparations.

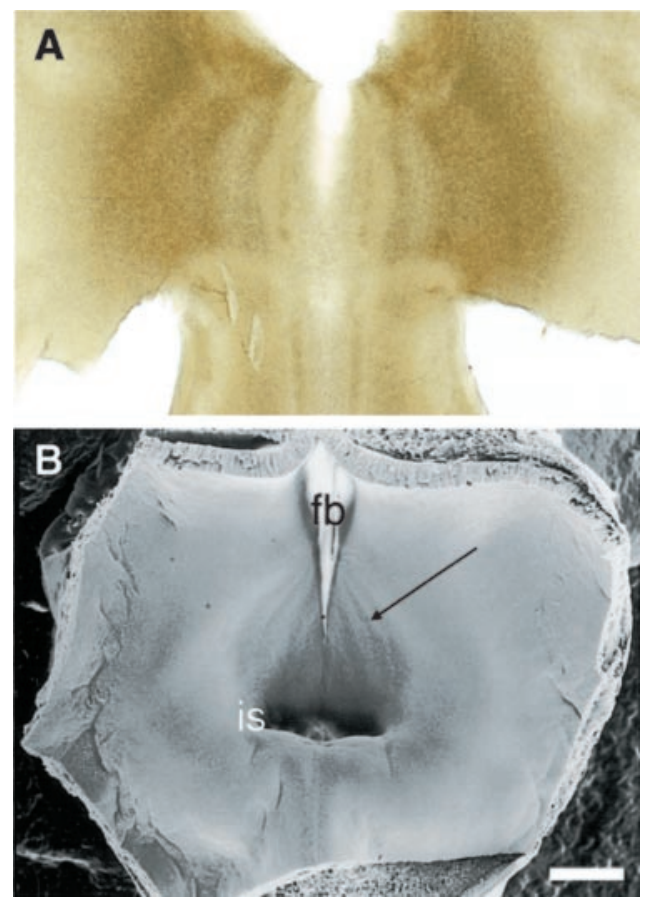

Figure 6. Arcs of the embryonic midbrain tegmentum have gross morphological correlates. $A$, Dissected, fixed but unstained whole mount of E5 ventral midbrain shows arcuate periodicities under transmitted light illumination. $B$, Scanning electron micrograph of a stage $26 / 27$ chick midbrain demonstrates a series of arcuate ridges, one of which is marked by an arrow, along the ventricular surface of the ventral midbrain. The dorsal half of the isthmus (is) is intact, but the view is otherwise as in Figure 1. fb, Forebrain. Scale bar, $250 \mu \mathrm{m}$.

et al., 1995; Schmidt-Ott et al., 1995). Among these genes, tailless $(t l l)$ is required for the development of the protocerebrum, the most anterodorsal brain region, whereas orthodenticle regulates an overlapping domain that includes part of the protocerebrum and extends into the adjoining brain region, the deuterocerebrum (Younossi-Hartenstein et al., 1997). We reexamined the expression patterns of TAILLESS/NR2E1, the only reported vertebrate homolog of tll (Yu et al., 1994; Monaghan et al., 1995), and the orthodenticle homolog OTX2 (Simeone et al., 1992), in light of our finding of arcs in embryonic midbrain and forebrain. As is true of their homologs in flies, TAILLESS is expressed throughout the most anterodorsal part of embryonic brain, and the OTX2 expres-
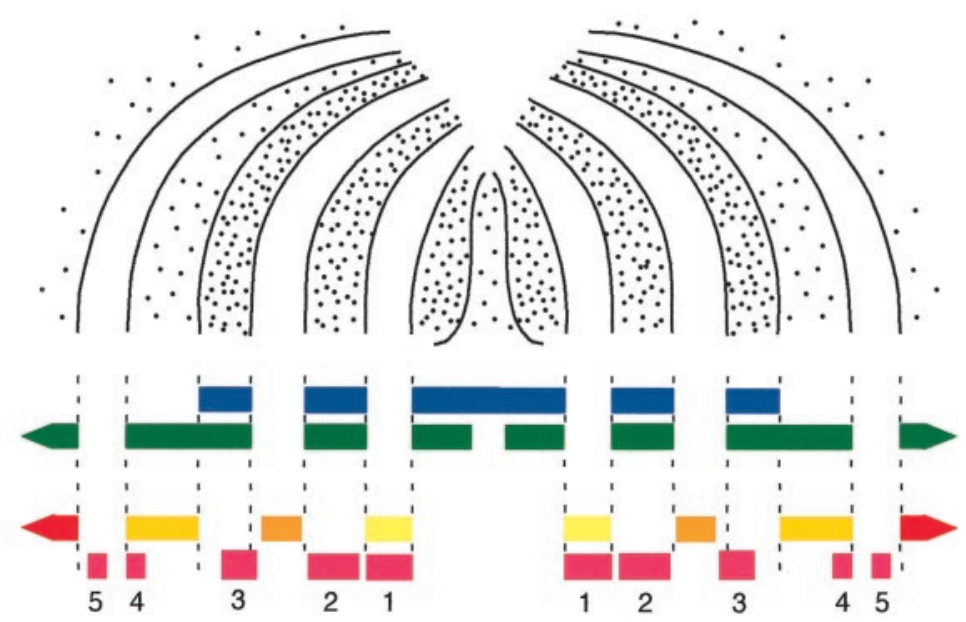

sion domain overlaps that of TAILLESS (Fig. 7C,D). The posterior border of $O T X 2$ expression coincides with the midbrainhindbrain junction (Fig. 5C) (Millet et al., 1996). The TAILLESS domain does not, however, correspond to any classic division of brain, such as forebrain. Instead, TAILLESS gene expression identifies a specific domain in the midbrain and forebrain: the dorsal midbrain and forebrain dorsal and anterior to the subthalamic region. Thus, arcs and TAILLESS gene expression together divide midbrain and forebrain into two nonoverlapping domains: the TAILLESS-negative arcs of midbrain and subthalamic tegmentum and a TAILLESS-positive cap comprising midbrain tectum and nonsubthalamic forebrain.

\section{DISCUSSION}

Three lines of evidence establish that the fundamental embryonic plan of the ventral midbrain is a series of arcuate territories arrayed bilateral to the ventral midline. First, this organization is macroscopically visible in dissected brain as refractive differences in whole-mount histology and as longitudinal ridges along the ventricular surface. Second, the neurons in the ventral midbrain, as demonstrated by histochemistry for the neuronal markers AChE and class III $\beta$-tubulin, are not homogeneously distributed across the mantle layer but are arrayed into arcs. Third, in the midbrain ventricular layer, arcuate periodicities in midbrain progenitor cells are detected by gene expression for developmental regulatory molecules, including ligands for $\mathrm{NOTCH}$ and WNT signaling pathways and the proneural gene $C A S H 1$. The finding of spatial registration among the arcuate patterns we observed shows that a common arcuate plan underlies the cellular and molecular organization of the embryonic midbrain tegmentum. More particularly, the three-dimensional registration of the ventricular layer periodicities and the mantle layer arcs strongly suggests a developmental plan in which the arcuate periodicities of the midbrain tegmentum are initiated among the progenitor cells of the ventricular layer, and the initial migration of postmitotic neurons from the ventricular layer into the mantle layer is radial. Radial migration away from the ventricular layer has been described in many regions of the brain, including the dorsal midbrain (Rakic, 1971, 1988; Gray and Sanes, 1991). In ventral midbrain, strict radial migration would account for the registration we found and provides for a straightforward mechanism preserving an arcuate distribution of cells as they travel from the ventricular layer into the mantle layer. 


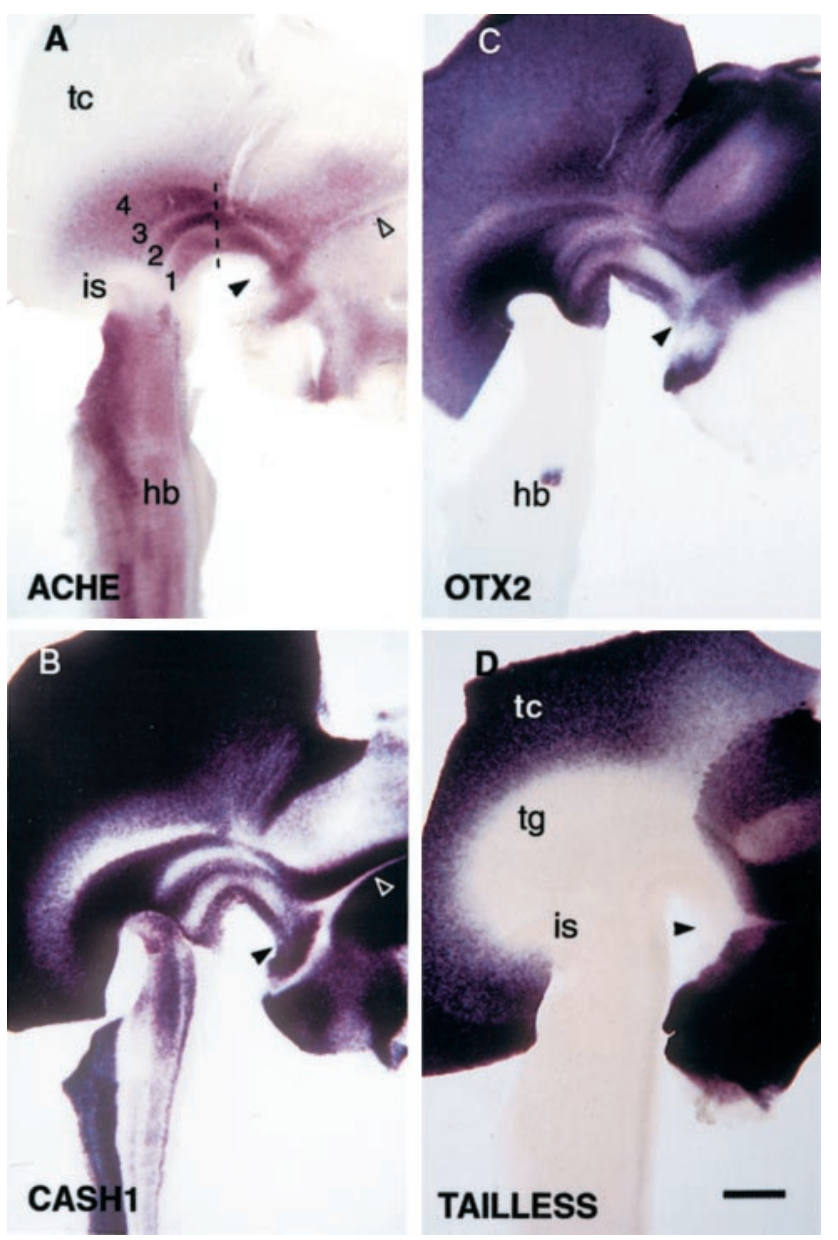

Figure 7. The embryonic chick brain rostral to the isthmus comprises two domains: one of the arcs aligned with the ventral midline $(A-C)$ and the other enriched in the gap gene homolog TAILLESS $(D)$. Illustrated are left half brains of stage 26 embryos, processed for whole-mount in situ hybridization and mounted for a sagittal view from the ventricular surface. The cephalic flexure places the hindbrain in the bottom left quadrant of each panel, the ventral midbrain near the center, and the forebrain to the right. $A, A C H E$-rich arcs of the midbrain tegmentum extend into the subthalamic region of forebrain. The midbrain-forebrain junction that the arcs traverse is marked by a dotted line. The position of the rostral limit of the arcs in subthalamus is noted by a filled arrowhead $(A-D)$, and the filled arrowhead is pointed toward the zli, which is indicated by an open arrowhead in $A$ and $B$. B. Arcuate stripes in midbrain ventricular layer, here demonstrated by expression of the proneural gene $C A S H 1$, also continue into the embryonic subthalamus. In rostral subthalamus, the lateral $C A S H 1$ bands appear not to stop but to deflect from their arcuate course to align with the zli. This trajectory may reflect that of Sonic Hedgehog, which in rostral subthalamus withdraws from the ventral midline and tracks the zli dorsally (Ericson et al., 1995; Marti et al., 1995). $C, D$, Gap gene homologs OTX2 and TAILLESS identify overlapping expression domains in the ventricular layer of rostral embryonic brain. The arc domain of midbrain and subthalamic tegmentum $(A)$ is enriched in OTX2 expression $(C)$ but is almost free of TAILLESS expression $(D)$. The OTX2 hybridization also demonstrates arcuate heterogeneities in ventral midbrain ventricular layer and an isolated clump of $O T X 2$-positive cells at the caudal end of rhombomere 1 in hindbrain. 1-4, Arcs 1-4; $h b$, hindbrain; $i s$, isthmus; $t c$, midbrain tectum; $t g$, tegmentum. Scale bar: $A-C$, $500 \mu \mathrm{m} ; D, 440 \mu \mathrm{m}$.

\section{Midbrain nucleogenesis}

For hindbrain and spinal cord development, it is accepted that particular neuronal cell types, such as motor neurons, are specified initially in longitudinal columns and subsequently migrate to take up their correct position in mature CNS (Harkmark, 1954; Tan and Le Douarin, 1991; Hemond and Glover, 1993; Clarke et al., 1998). For motor neurons and secondary sensory neurons in hindbrain, these migratory cell populations, as they form distinct nuclei, retain their organization into longitudinal cell columns. In contrast, the nuclei of the mature midbrain are not described as longitudinal columns, the exception being the oculomotor complex, which we found to arise in the first arc. Our data, however, show that the midbrain arcs differ in their molecular identities, suggesting the general hypothesis that the arcs and interarcs act as staging territories for assigning neurons to nuclear fates. What then becomes of this arcuate plan in mature midbrain? One possibility is that the arcuate territories migrate, as do columns in hindbrain, but lose their longitudinal organization and acquire different shapes. A second possibility is that there is a covert organization of at least parts of midbrain tegmentum into longitudinal zones. Recent work on the midbrain periaqueductal gray, which appears fairly homogeneous in Nissl-stained material, suggests that this structure may in fact be functionally organized into longitudinal columns (Bandler and Shipley, 1994). Fate-mapping studies of the arcs, combined with a reexamination of adult midbrain systems neuroanatomy, will be needed to address the specific relationships between arcs and their descendents.

\section{Arcs extend into the subthalamus}

On the basis of fiber-connection data and histology, neuroanatomists since Forel (1877) have viewed the subthalamus of the adult vertebrate forebrain as a rostral extension of midbrain tegmentum (Kuhlenbeck, 1939; Herrick, 1948; Nauta and Haymaker, 1969). Our finding that midbrain arcs continue rostrally into the subthalamic region indicates that there may be an embryological basis for this similarity. Indeed, one view might be that the subthalamic tegmentum is simply displaced midbrain tegmentum. Both classical and recent models of vertebrate brain embryology, however, place the territory of the subthalamic arcs within diencephalon (Herrick, 1948; Figdor and Stern, 1993; Rubenstein et al., 1994). Moreover, midbrain and diencephalic arc patterns are clearly distinct. At the transition from midbrain to forebrain, the number of arcs is abruptly reduced, and of the midbrain homeobox genes $P H O X 2 A, P A X 6$, and $E V X 1$, only $P A X 6$ is expressed in the subthalamus (data not illustrated). These observations indicate that the midbrain and subthalamic arcs share the property of being columns of neurons aligned with the ventral midline but differ in their cell-type composition.

\section{Arcs and the epichordal nervous system}

The arcuate plan of the midbrain and subthalamic tegmentum shares many features with the longitudinal organization of the embryonic hindbrain and spinal cord. Most prominently, both tissues contain within their mantle layers columns of neurons aligned with the ventral midline (this study; Kallen, 1962; Chitnis et al., 1995) and within their ventricular layers interdigitating stripes of gene expression for the NOTCH ligands DELTA and SERRATE (this study; Lindsell et al., 1996; Myat et al., 1996; Matise and Joyner, 1997). Moreover, just as ventricular ridges identify the arcs of the midbrain, so too do ventricular ridges reflect the presence of longitudinal cell columns in the vertebrate hindbrain, being evident in the embryos of many species and particularly prominent in adult fish (Herrick, 1915). At the molecular level, there are many details of gene expression that differ between the midbrain arcs and the columns of caudal CNS. For example, $P A X 6$ gene expression in ventral midbrain identifies a 


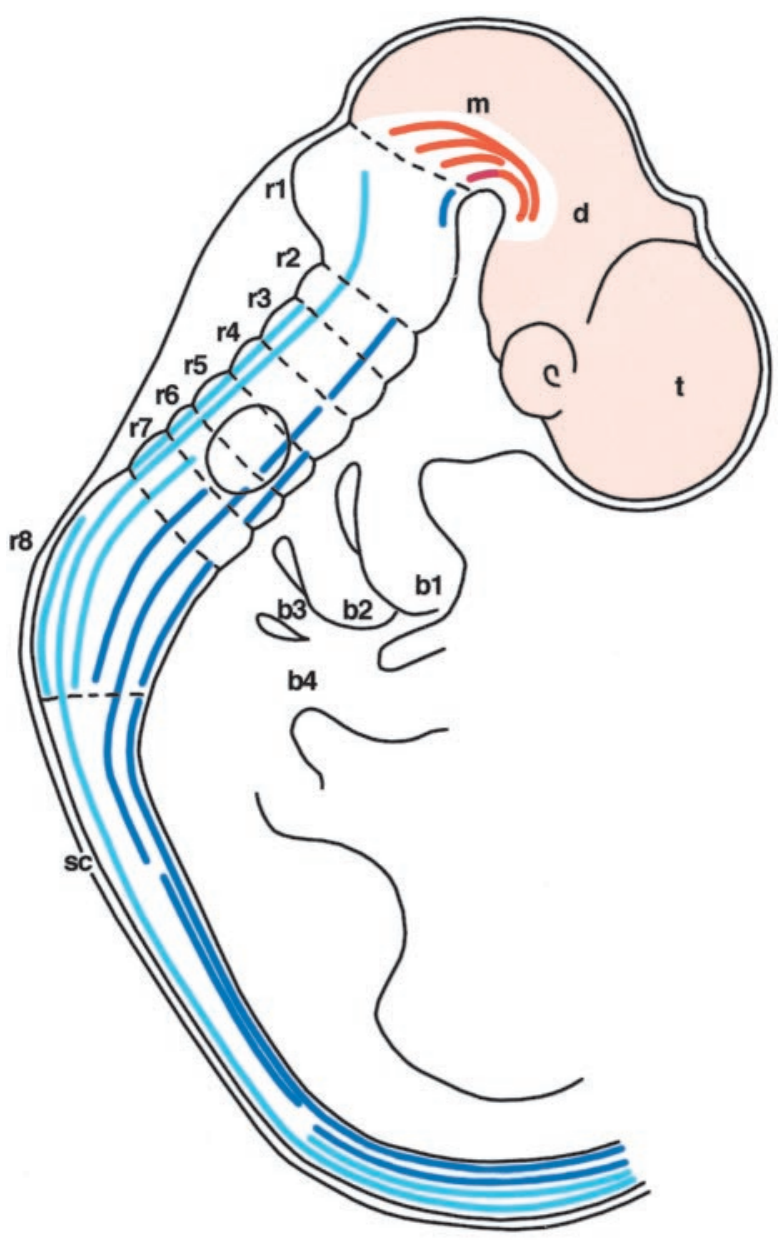

Figure 8. Diagram of a chick embryo illustrating the proposed division of vertebrate embryonic CNS into the following: (1) a neuraxial region containing multiple neuronal columns, including arcs, aligned with the ventral midline and (2) an anterodorsal cap distinguished by its expression of the gap gene homolog TAILLESS. The columns illustrated identify midbrain and subthalamic arcs (red), secondary sensory neurons (light blue), and motor neurons (dark blue). The TAILLESS cap (beige) consists of dorsal midbrain and nonsubthalamic forebrain. Chick embryo sketch adapted from Lumsden (1990). b1-b4, Branchial arches 1-4; $d$, diencephalon; $m$, midbrain; $r 1-r 8$, rhombomeres $1-8$; $s c$, spinal cord; $t$, telencephalon.

restricted territory in the mantle layer clearly lateral to the motor neurons of arc 1, but in hindbrain and spinal cord, $P A X 6$ is expressed in a broad domain of the ventricular layer, including the progenitor territory for some motor neurons (Jessell, 2000). Nonetheless, at a synoptic level, our finding of an arcuate plan to midbrain and subthalamus suggests a fundamental division of embryonic CNS into the following: (1) a neuraxis from spinal cord to subthalamus containing frank cell columns, including arcs, aligned with the ventral midline and (2) an anterodorsal cap distinguished by gene expression for TAILLESS (Fig. 8).

It seems very likely that such an anterior-posterior division in CNS reflects anterior-posterior differences in the signaling properties of the ventral midline and underlying axial mesoderm. The classical floor plate of Kingsbury (1922) is restricted to spinal cord and hindbrain, but more recent authors identify a rostral floor plate in ventral midbrain and caudal diencephalon on histological and gene expression grounds (Kuhlenbeck, 1973; Dale et al., 1997; Pera and Kessel, 1997). The relationship of the rostral floor plate to underlying axial mesoderm is not clear because of the tremendous tissue movements that take place in early embryogenesis (Vaage, 1969; Dale et al., 1999). However, detailed molecular embryological studies, including work in explant culture, indicate that the critical interactions that produce midbrain floor plate involve the underlying notochord, whereas the prechordal mesendoderm mediates the induction of the rostral diencephalic ventral midline, which is molecularly distinct from floor plate (Dale et al., 1997, 1999; Foley et al., 1997; Pera and Kessel, 1997). Together, these observations suggest that one embryological basis for the difference between the neuraxis of columns and arcs and the TAILLESS-rich anterodorsal cap is that the neuraxis is epichordal and has a floor plate, whereas the ventral midline of the cap overlies prechordal mesendoderm (Puelles et al., 1987; Shimamura et al., 1995; Foley et al., 1997; Dale et al., 1999).

One conspicuous feature of the floor plate in spinal cord and hindbrain and its rostral extension into midbrain and subthalamus is that it expresses the signaling molecule Sonic Hedgehog (SHH). Does ventral midline SHH, known to regulate cell-type specification in ventral spinal cord (Jessell, 2000), also control ventral midbrain patterning? We found recently that ectopic $\mathrm{SHH}$ in midbrain can regulate the expression pattern of three homeobox genes, $P H O X 2 A, P A X 6$, and EVX1 (Agarwala et al., 2001), which serve to mark the midbrain arcs (this study). Whether SHH can function more globally to elicit the complete program of ventral midbrain development, including the generation of ventricular layer periodicities and the formation of mature midbrain nuclei, remains to be established.

\section{TAILLESS gene expression in rostral brain}

Immense molecular insight into hindbrain embryology has issued from the modern rediscovery of rhombomeres, the cellular evidence that rhombomeres form neurectoderm segments, and the genetic evidence that specific Drosophila HOM-C homologs control features of rhombomere identity (Lumsden and Krumlauf, 1996). In contrast, relatively few similarities in detailed structure have been identified between the anterior ends of the brains of insects and vertebrates. The (supraesophageal) insect brain comprises the protocerebrum, the deuterocerebrum, and the tritocerebrum. There is broad acceptance in the literature that the deuterocerebrum and tritocerebrum, both of which express HOM-C genes (Hirth et al., 1998), are modified segments. The segmental status of the most anterodorsal region, the protocerebrum, remains, however, as controversial in the insect literature as do claims in the vertebrate literature for a segmental organization to the forebrain (Finkelstein and Perrimon, 1991; Rubenstein et al., 1994; Schmidt-Ott et al., 1995; Younossi-Hartenstein et al., 1996; Larsen et al., 2001).

Our findings suggest a different purchase on the problem of how rostral brain organization relates to that of more caudal CNS. Younossi-Hartenstein et al. (1997) have described in detail the pattern of gap gene expression in the neuroblasts that give rise to the fly larval brain. The protocerebrum is specifically identified by gene expression for the terminal gap gene $t l l$, whereas the head gap gene orthodenticle is expressed in an overlapping domain of posterior protocerebrum and the anterior half of the deuterocerebrum. Gene isolation and characterization studies in chick and mouse embryos showed that the vertebrate $t l l$ homolog is expressed in anterior CNS (Yu et al., 1994; Monaghan et al., 1995), but its expression domain, forebrain and dorsal midbrain, did not appear to correspond to any known structural division within vertebrate brain. We have now identified a morphological correlate for the TAILLESS expression domain in 
chick brain. The arc-containing ventral midbrain and subthalamus are TAILLESS negative; the rest of the midbrain and forebrain is enriched in TAILLESS gene expression. This finding, that the homolog of a Drosophila protocerebrum marker identifies a novel division of chick brain, suggests that fly protocerebrum and vertebrate rostral brain will show additional similarities of morphology and gene regulation (Chang et al., 2001; Reichert and Simeone, 2001; Page, 2002). Moreover, our finding that the midbrain and subthalamic tegmentum share with more caudal CNS a common embryological plan of cell columns aligned with the ventral midline predicts that the $t l$-positive protocerebrum will differ from ventral nerve cord and caudal fly brain in features of its longitudinal organization (Arendt and Nubler-Jung, 1999).

\section{REFERENCES}

Agarwala S, Sanders TA, Ragsdale CW (2001) Sonic Hedgehog control of size and shape in midbrain pattern formation. Science 291:2147-2150.

Arendt D, Nubler-Jung K (1999) Comparison of early nerve cord development in insects and vertebrates. Development 126:2309-2325.

Bally-Cuif L, Wassef M (1994) Ectopic induction and reorganization of Wnt-1 expression in quail/chick chimeras. Development 120:3379-3394. Bally-Cuif L, Gulisano M, Broccoli V, Boncinelli E (1995) c-otx2 is expressed in two different phases of gastrulation and is sensitive to retinoic acid treatment in chick embryo. Mech Dev 49:49-63.

Bandler R, Shipley MT (1994) Columnar organization in the midbrain periaqueductal gray: modules for emotional expression? Trends Neurosci 17:379-389.

Chang T, Mazotta J, Dumstrei K, Dumitrescu A, Hartenstein V (2001) Dpp and Hh signaling in the Drosophila embryonic eye field. Development 128:4691-4704.

Chitnis A, Henrique D, Lewis J, Ish-Horowicz D, Kintner C (1995) Primary neurogenesis in Xenopus embryos regulated by a homologue of the Drosophila neurogenic gene Delta. Nature 375:761-766.

Clarke JD, Erskine L, Lumsden A (1998) Differential progenitor dispersal and the spatial origin of early neurons can explain the predominance of single-phenotype clones in the chick hindbrain. Dev Dyn 212:14-26.

Coggeshall RE (1964) A study of diencephalic development in the albino rat. J Comp Neurol 122:241-269.

Cohen SM, Jurgens G (1990) Mediation of Drosophila head development by gap-like segmentation genes. Nature 346:482-485.

Dale JK, Vesque C, Lints TJ, Sampath TK, Furley A, Dodd J, Placzek M (1997) Cooperation of BMP7 and SHH in the induction of forebrain ventral midline cells by prechordal mesoderm. Cell 90:257-269.

Dale JK, Sattar N, Heemskerk J, Clarke JDW, Placzek M, Dodd J (1999) Differential patterning of ventral midline cells by axial mesoderm is regulated by BMP7 and chordin. Development 126:397-408.

Dealy CN, Roth A, Ferrari D, Brown AM, Kosher RA (1993) Wnt-5a and Wnt-7a are expressed in the developing chick limb bud in a manner suggesting roles in pattern formation along the proximodistal and dorsoventral axes. Mech Dev 43:175-186.

Easter Jr SS, Ross LS, Frankfurter A (1993) Initial tract formation in the mouse brain. J Neurosci 13:285-299.

Ericson J, Muhr J, Placzek M, Lints T, Jessell TM, Edlund T (1995) Sonic hedgehog induces the differentiation of ventral forebrain neurons: a common signal for ventral patterning within the neural tube. Cell 81:747-756.

Ernsberger U, Patzke H, Tissier-Seta JP, Reh T, Goridis C, Rohrer H (1995) The expression of tyrosine hydroxylase and the transcription factors cPhox-2 and Cash-1: evidence for distinct inductive steps in the differentiation of chick sympathetic precursor cells. Mech Dev $52: 125-136$.

Figdor MC, Stern CD (1993) Segmental organization of embryonic diencephalon. Nature 363:630-634.

Finkelstein R, Perrimon N (1991) The molecular genetics of head development in Drosophila melanogaster. Development 112:899-912.

Foley AC, Storey KG, Stern CD (1997) The prechordal region lacks neural inducing ability, but can confer anterior character to more posterior neuroepithelium. Development 124:2983-2996.

Forel A (1877) Untersuchungen uber die haubenregion und ihre oberen verknupf ungen im gehirn des menschen und einiger saugetiere, mit beitragen zu den methoden der gehirnuntersuchungen. Arch Psychiat 7:393-495.

Goulding MD, Chalepakis G, Deutsch U, Erselius JR, Gruss P (1991) Pax-3, a novel murine DNA binding protein expressed during early neurogenesis. EMBO J 10:1135-1147.

Goulding MD, Lumsden A, Gruss P (1993) Signals from the notochord and floor plate regulate the region-specific expression of two Pax genes in the developing spinal cord. Development 117:1001-1016.

Gray GE, Sanes JR (1991) Migratory paths and phenotypic choices of clonally related cells in the avian optic tectum. Neuron 6:211-225.

Graybiel AM, Ragsdale CW (1978) Histochemically distinct compartments in the striatum of human, monkeys, and cat demonstrated by acetylthiocholinesterase staining. Proc Natl Acad Sci USA 75:5723-5726.

Grove EA, Tole S, Limon J, Yip L, Ragsdale CW (1998) The hem of the embryonic cerebral cortex is defined by the expression of multiple Wnt genes and is compromised in Gli3-deficient mice. Development 125:2315-2325

Hamburger V, Hamilton HL (1951) A series of normal stages in the development of the chick embryo. J Morphol 88:49-92.

Harkmark W (1954) Cell migrations from the rhombic lip to the inferior olive, the nucleus raphe and the pons: a morphological and experimental investigation on chick embryos. J Comp Neurol 100:115-209.

Hayashi H, Mochii M, Kodama R, Hamada Y, Mizuno N, Eguchi G, Tachi C (1996) Isolation of a novel chick homolog of Serrate and its coexpression with C-Notch-1 in chick development. Int J Dev Biol 40:1089-1096.

Hemond SG, Glover JC (1993) Clonal patterns of cell proliferation, migration, and dispersal in the brainstem of the chicken embryo. J Neurosci 13:1387-1402.

Herrick CJ (1915) An introduction to neurology. Philadelphia: Saunders.

Herrick CJ (1948) The brain of the tiger salamander. Chicago: University of Chicago.

Hirth F, Therianos S, Loop T, Gehring WJ, Reichert H, FurukuboTokunaga K (1995) Developmental defects in brain segmentation caused by mutations of the homeobox genes orthodenticle and empty spiracles in Drosophila. Neuron 15:769-778.

Hirth F, Hartmann B, Reichert H (1998) Homeotic gene action in embryonic brain development of Drosophila. Development 125:1579-1589.

His W (1904) Die entwicklung des menschlichen gehirns wahrend der ersten monate. Leipzig, Germany: Hirzel.

Hugosson R (1958) Morphologic and experimental studies on the development and significance of the rhombencephalic longitudinal cell columns. PhD thesis, University of Lund.

Jasoni CL, Walker MB, Morris MD, Reh TA (1994) A chicken achaetescute homolog (CASH-1) is expressed in a temporally and spatially discrete manner in the developing nervous system. Development 120:769-783

Jessell TM (2000) Neuronal specification in the spinal cord: inductive signals and transcriptional codes. Nat Rev Genet 1:20-29.

Johnston JB (1906) The nervous system of vertebrates. Philadelphia: Blakiston.

Johnston JB (1923) Further contributions to the study of the evolution of the forebrain. V. Survey of forebrain morphology. J Comp Neurol 36:143-192.

Kallen B (1962) Mitotic patterning in the central nervous system of chick embryos; studied by a colchicine method. Z Anat Entwicklungsgesch 123:309-319.

Kingsbury BF (1922) The fundamental plan of the vertebrate brain. J Comp Neurol 34:461-491.

Kuhlenbeck H (1939) The development and structure of the pretectal cell masses in the chick. J Comp Neurol 71:361-387.

Kuhlenbeck H (1973) The central nervous system of vertebrates, Vol 3, Pt II, Overall morphologic pattern. Basel: Karger.

Larsen CW, Zeltser LM, Lumsden A (2001) Boundary formation and compartition in the avian diencephalon. J Neurosci 21:4699-4711.

Layer PG (1983) Comparative localization of acetylcholinesterase and pseudocholinesterase during morphogenesis of the chicken brain. Proc Natl Acad Sci USA 80:6413-6417.

Lee MK, Tuttle JB, Rebhun LI, Cleveland DW, Frankfurter A (1990) The expression and posttranslational modification of a neuron-specific beta-tubulin isotype during chick embryogenesis. Cell Motil Cytoskeleton 17:118-132.

Lindsell CE, Boulter J, diSibio G, Gossler A, Weinmaster G (1996) Expression patterns of Jagged, Delta1, Notch1, Notch2, and Notch3 genes identify ligand-receptor pairs that may function in neural development. Mol Cell Neurosci 8:14-27.

Logan C, Wingate RJ, McKay IJ, Lumsden A (1998) Tlx-1 and Tlx-3 homeobox gene expression in cranial sensory ganglia and hindbrain of the chick embryo: markers of patterned connectivity. J Neurosci 18:5389-5402.

Lopata MA, Havercroft JC, Chow LT, Cleveland DW (1983) Four unique genes required for beta tubulin expression in vertebrates. Cell 32:713-724

Lumsden A (1990) The cellular basis of segmentation in the developing hindbrain. Trends Neurosci 13:329-335.

Lumsden A, Krumlauf R (1996) Patterning the vertebrate neuraxis. Science 274:1109-1115.

Marti E, Takada R, Bumcrot DA, Sasaki H, McMahon AP (1995) Distribution of Sonic hedgehog peptides in the developing chick and mouse embryo. Development 121:2537-2547. 
Matise MP, Joyner AL (1997) Expression patterns of developmental control genes in normal and Engrailed-1 mutant mouse spinal cord reveal early diversity in developing interneurons. J Neurosci 17:7805-7816.

Menezes JR, Luskin MB (1994) Expression of neuron-specific tubulin defines a novel population in the proliferative layers of the developing telencephalon. J Neurosci 14:5399-5416.

Millet S, Bloch-Gallego E, Simeone A, Alvarado-Mallart RM (1996) The caudal limit of Otx2 gene expression as a marker of the midbrain/ hindbrain boundary: a study using in situ hybridisation and chick/quail homotopic grafts. Development 122:3785-3797.

Monaghan AP, Grau E, Bock D, Schutz G (1995) The mouse homolog of the orphan nuclear receptor tailless is expressed in the developing forebrain. Development 121:839-853.

Moody SA, Stein DB (1988) The development of acetylcholinesterase activity in the embryonic nervous system of the frog, Xenopus laevis. Brain Res 467:225-232.

Moody SA, Quigg MS, Frankfurter A (1989) Development of the peripheral trigeminal system in the chick revealed by an isotype-specific anti-beta-tubulin monoclonal antibody. J Comp Neurol 279:567-580.

Myat A, Henrique D, Ish-Horowicz D, Lewis J (1996) A chick homologue of Serrate and its relationship with Notch and Delta homologues during central neurogenesis. Dev Biol 174:233-247.

Nauta WJH, Feirtag M (1986) Fundamental neuroanatomy. New York: Freeman.

Nauta WJH, Haymaker W (1969) Hypothalamic nuclei and fiber connections. In: The hypothalamus (Haymaker W, Anderson E, Nauta WJH, eds), pp 136-209. Springfield, IL: Thomas.

Page DT (2002) Inductive patterning of the embryonic brain in Drosophila. Development 129:2121-2128.

Parr BA, Shea MJ, Vassileva G, McMahon AP (1993) Mouse Wnt genes exhibit discrete domains of expression in the early embryonic CNS and limb buds. Development 119:247-261.

Pattyn A, Morin X, Cremer H, Goridis C, Brunet J-F (1997) Expression and interactions of two closely related homeobox genes Phox $2 \mathrm{a}$ and Phox $2 b$ during neurogenesis. Development 124:4065-4075.

Paxinos G, Watson C (1986) The rat brain in stereotaxic coordinates, Ed 2. Sydney: Academic.

Pera EM, Kessel M (1997) Patterning of the chick forebrain anlage by the prechordal plate. Development 124:4153-4162.

Puelles L, Amat JA, Martinez-de-la-Torre M (1987) Segment-related, mosaic neurogenetic pattern in the forebrain and mesencephalon of early chick embryos. I. Topography of AChE-positive neuroblasts up to stage HH18. J Comp Neurol 266:247-268.

Rakic P (1971) Neuron-glia relationship during granule cell migration in developing cerebellar cortex. A Golgi and electronmicroscopic study in Macacus rhesus. J Comp Neurol 141:283-312.
Rakic P (1988) Specification of cerebral cortical areas. Science 241:170-176.

Reichert H, Simeone A (2001) Developmental genetic evidence for a monophyletic origin of the bilaterian brain. Philos Trans R Soc Lond B Biol Sci 356:1533-1544.

Rose JE (1942) The ontogenetic development of the rabbit's diencephalon. J Comp Neurol 77:61-129.

Ross LS, Parrett T, Easter Jr SS (1992) Axonogenesis and morphogenesis in the embryonic zebrafish brain. J Neurosci 12:467-482.

Rubenstein JL, Martinez S, Shimamura K, Puelles L (1994) The embryonic vertebrate forebrain: the prosomeric model. Science 266:578-580.

Schmidt-Ott U, Gonzalez-Gaitan M, Technau GM (1995) Analysis of neural elements in head-mutant Drosophila embryos suggests segmental origin of the optic lobes. Rouxs Arch Dev Biol 205:31-44.

Schulte HvW, Tilney F (1915) Development of the neuraxis in the domestic cat to the stage of twenty-one somites. Ann NY Acad Sci 24:319-346.

Shimamura K, Hartigan DJ, Martinez S, Puelles L, Rubenstein JL (1995) Longitudinal organization of the anterior neural plate and neural tube. Development 121:3923-3933.

Sidman RL, Rakic P (1982) Development of the human central nervous system. In: Histology and histopathology of the nervous system (Haymaker W, Adams RD, eds), pp 3-145. Springfield, IL: Thomas.

Simeone A, Acampora D, Gulisano M, Stornaiuolo A, Boncinelli E (1992) Nested expression domains of four homeobox genes in developing rostral brain. Nature 358:687-690.

Tan K, Le Douarin NM (1991) Development of the nuclei and cell migration in the medulla oblongata. Application of the quail-chick chimera system. Anat Embryol 183:321-343.

Tsuchida T, Ensini M, Morton SB, Baldassare M, Edlund T, Jessell TM, Pfaff SL (1994) Topographic organization of embryonic motor neurons defined by expression of LIM homeobox genes. Cell 79:957-970.

Vaage S (1969) The segmentation of the primitive neural tube in chick embryos (Gallus domesticus). A morphological, histochemical and autoradiographical investigation. Ergeb Anat Entwicklungsgesch 41:3-87.

Yamada T, Pfaff SL, Edlund T, Jessell TM (1993) Control of cell pattern in the neural tube: motor neuron induction by diffusible factors from notochord and floor plate. Cell 73:673-686.

Younossi-Hartenstein A, Nassif C, Green P, Hartenstein V (1996) Early neurogenesis of the Drosophila brain. J Comp Neurol 370:313-329.

Younossi-Hartenstein A, Green P, Liaw GJ, Rudolph K, Lengyel J, Hartenstein V (1997) Control of early neurogenesis of the Drosophila brain by the head gap genes tll, otd, ems, and btd. Dev Biol 182:270-283.

Yu RT, McKeown M, Evans RM, Umesono K (1994) Relationship between Drosophila gap gene tailless and a vertebrate nuclear receptor Tlx. Nature 370:375-379. 